\title{
LNC00II 5 Mediates Cisplatin Resistance by Regulating the miR-7/ERK Signalling Pathway in Ovarian Cancer
}

\author{
Xinyan Jiang \\ Yan Cheng \\ Yanan $\mathrm{He}$ \\ Shanshan Cong \\ Liyuan Sun \\ Di Wu \\ Han Wu \\ Guangmei Zhang
}

The First Affiliated Hospital, Harbin Medical University, Harbin, People's Republic of China
Correspondence: Guangmei Zhang Building 2, 23 Youzheng Road, Harbin, I5000I, People's Republic of China Email guangmeizhang@I26.com
Background: Ovarian cancer has one of the highest mortality rates among all gynaecological malignancies, and increasing evidence suggests that lncRNAs are widely involved in the development of ovarian tumours. This study aimed to investigate the mechanism of the LNC00115/miR-7/ERK axis in the cisplatin resistance of ovarian cancer cells.

Methods: The expression of miR-7 and LNC00115 in ovarian cancer cell lines and tissues was detected by qRT-PCR. The ovarian cancer cell lines were constructed by overexpressing or knocking down the expression of LNC00115 or miR-7. CCK-8, transwell invasion, Western blot, immunohistochemistry, and luciferase reporter assays were carried out to identify the targets of LNC00115 and explore its roles and mechanisms in ovarian cancer. A nude mouse model was established, and the expression of LNC00115, miR-7 and ERK was detected. The changes in the tumours and body weights of the nude mice were measured.

Results: LNC00115 was upregulated in ovarian cancer tissues and cisplatin-resistant ovarian cancer cells. Moreover, LNC00115 promoted the cisplatin resistance, invasion and migration of ovarian cancer cells. LNC00115 was shown to directly target miR-7, and miR-7 was downregulated in ovarian cancer tissues and cisplatin-resistant ovarian cancer cells. miR-7 inhibited the cisplatin resistance, invasion and migration of ovarian cancer cells and directly targeted ERK. ERK was overexpressed in cisplatin-resistant ovarian cancer cells and ovarian cancer tissues. In animal experiments, overexpression of LNC00115 enhanced the cisplatin resistance of ovarian cancer cells, while miR-7 had the opposite effect. Mechanistically, LNC00115 sponged miR-7 to increase the expression of ERK, which in turn enhanced the cisplatin resistance of ovarian cancer.

Conclusion: Our data clarify the mechanism by which the LNC00115/miR-7/ERK axis promotes cisplatin resistance and provide a new clinical strategy for combating cisplatin resistance in ovarian cancer.

Keywords: cisplatin resistance, miR-7, LNC00115, ovarian cancer, ERK

\section{Introduction}

Ovarian cancer is one of the most common gynaecological malignancies and has the highest mortality rate among these tumours. ${ }^{1}$ The first-line therapies for ovarian cancer include surgery and cisplatin-based chemotherapy. However, cisplatin resistance has become one of the most important obstacles to the successful treatment of patients with ovarian cancer. ${ }^{2}$ Little is known about the early diagnosis of ovarian cancer or the mechanism of drug resistance. 
Long noncoding RNAs (lncRNAs) have transcripts that are longer than $200 \mathrm{nt}$ and were originally considered to be "noise" in genomic transcription. ${ }^{3}$ lncRNAs can interact directly or indirectly with micro RNAs (miRNAs), resulting in the loss of regulatory function. ${ }^{4}$ Increased understanding of lncRNAs has revealed important regulatory roles in processes related to gene expression, including tumourigenesis, ${ }^{5}$ development, ${ }^{6}$ metastasis ${ }^{7}$ and drug resistance. ${ }^{8}$ More importantly, lncRNAs are considered to be key regulators of tumour drug resistance and may be important targets for predicting prognosis and evaluating therapeutic effects. ${ }^{9}$

miRNAs are widely involved in physiological and pathological processes, play an important role in gene regulatory networks ${ }^{10}$ and are regulated by the overall biological regulatory network. miR-7 is related to the occurrence and development of a variety of cancers and mainly functions as a tumour suppressor. ${ }^{11}$ An increasing number of studies have shown that miR-7 can reverse chemotherapy resistance. ${ }^{12,13}$ For example, miR-7 can reverse paclitaxel/carboplatin resistance in breast cancer by inhibiting MRP1 and BCL2.

In this study, we analysed the effects of LNC00115 and miR-7 on cisplatin resistance in ovarian cancer and confirmed that LNC00115 plays a carcinogenic role in migration and invasion. The purpose of this study was to explore the diagnostic and therapeutic value of LNC00115/miR-7/ ERK as well as the molecular mechanism in the context of cisplatin resistance in ovarian cancer.

\section{Materials and Methods}

\section{Patient Specimens and Cell Lines}

The use of human tissue was approved by the ethics committee of Harbin Medical University First Affiliated Hospital. Tissue specimens were collected at the First Affiliated Hospital of Harbin Medical University. A total of 6 pairs of ovarian cancer tissues and adjacent tissues were obtained. The hospital's ethics committee and all patients provided informed consent, and the study was performed in accordance with the Helsinki Declaration. The human ovarian cancer cell lines SKOV3 and SKOV3-DDP were obtained from the First Affiliated Hospital of Harbin Medical University and were approved for use by the hospital ethics committee. SKOV3 cells were maintained in RPMI 1640 medium (HyClone, USA) supplemented with $10 \%$ foetal bovine serum (FBS; Ausbian, Austria) and 1\% penicillinstreptomycin at $37^{\circ} \mathrm{C}$ and $5 \% \mathrm{CO}_{2}$ in an incubator. The cisplatin-resistant cell line SKOV3-DDP was maintained in IMDM (HyClone, USA) supplemented with 10\% FBS and $1 \%$ penicillin-streptomycin at $37^{\circ} \mathrm{C}$ and $5 \% \mathrm{CO}_{2}$ in an incubator.

\section{Luciferase Reporter Assay}

Luciferase reporter assays were performed using the Luciferase Reporter Assay System (Promega Corporation, Fitchburg, WI, USA). Briefly, 293T cells were inoculated in a 12-well plate and cotransfected with the pmirGLO-ERK-WT, pmirGLO-ERK-MUT, pmirGLOLNC00115-WT or pmirGLO-LNC00115-MUT vector and the miR-7 mimics or miR-NC (GenePharma, Shanghai, China). After 48 hours, the firefly luciferase activity was measured and normalized to that of Renilla.

\section{miRNA Transfection}

The miR-7 mimics and inhibitors were synthesized by GenePharma (Shanghai, People's Republic of China). SKOV3 and SKOV3-DDP cells were seeded in six-well plates at $5 \times 10^{5}$ cells/well and then transfected with $2 \mu \mathrm{g}$ of the miR-7 mimics or miR-7 inhibitor using Lipofectamine $^{\circledR} 2000$ reagent (Thermo Fisher Scientific, USA). At 48 hours after transfection, the cells were selected with G418 (4 $\mu \mathrm{g} / \mathrm{mL}$; Gibco, USA) for one month, and GFP and Neo served as the selection markers for cells transfected with the miR-7 mimics and inhibitors. SKOV3 cells transfected with the miR-7 inhibitor are referred to as SKOV3-M cells, and SKOV3-DDP cells transfected with the miR-7 mimics are referred to as SKOV3-DDP-M cells.

\section{LncRNA Transfection}

The 1317-nucleotide LNC00115 sequence was cloned into the pCDNA3.1 $(+)$ vector to construct an LNC00115 overexpression vector (GenePharma Shanghai, People's Republic of China). For LNC00115 knockout, two RNAs directed at the LNC00115 promoter region were cloned into the pGPU6 vector (Gene Pharma Shanghai, People's Republic of China). Lipofectamine 2000 was used to transfect SKOV3 and SKOV3-DDP cells according to the manufacturer's instructions. At 48 hours after transfection, the cells were selected with puromycin $(2 \mu \mathrm{g} / \mathrm{mL})$ for one month, and stable cell lines with LNC00115 silencing or overexpression were thereby constructed. SKOV3 cells transfected with the LNC00115 overexpression vector are referred to as SKOV3-L cells, and SKOV3-DDP cells 
transfected with the LNC00115 silencing vector are referred to as SKOV3-DDP-L cells.

\section{Western Blot Analysis}

The cells were washed twice with PBS, lysed and incubated on ice for 1 hour. The cell lysate was centrifuged at $14,000 \mathrm{rpm}$ for 5 minutes, and the cell supernatant was collected. Aliquots of total protein $(20 \mu \mathrm{g})$ were separated by $12 \%$ sodium dodecyl sulfate polyacrylamide gel electrophoresis and transferred to a polyvinylidene fluoride membrane (EMD Millipore, Billerica, MA, USA). The membrane was blocked with 5\% BSA for 2 hours and then sequentially incubated overnight with a polyclonal rabbit anti-human p-ERK1/2 antibody (9101, Cell Signaling Technology, USA 1:200) and ERK1/2 antibody (Abclonal Technology, China $1: 200$ ) at $4^{\circ} \mathrm{C}$ and a secondary antibody (EMD Millipore, Burlington, MA, USA 1:1000) before detection of the proteins using a chemiluminescence detection system (Applygen, China). Images were obtained with a 5200 automatic ECL imaging system (Tanon, China).

\section{Cell Counting Kit-8 Assay}

Chemosensitivity was determined using a Cell Counting Kit-8 (CCK-8) assay (Dojindo Laboratories, Kumamoto, Japan). Briefly, $5 \times 10^{3}$ cells in $200 \mu \mathrm{L}$ of medium were plated into each well of a 96-well plate. When the cells reached confluence, $4 \mu \mathrm{g} / \mathrm{mL}$ cisplatin was added to the wells, and the cells were incubated for 48 hours. Then, 10 $\mu \mathrm{L}$ of CCK-8 reagent was added to each well, and the plates were incubated for 2-4 hours. Finally, the absorbance of each well at $450 \mathrm{~nm}$ was detected.

\section{Transwell Invasion Assay}

The invasion abilities of the cells were measured with invasion chambers containing filters with a pore diameter of $0.8 \mu \mathrm{m}$. The upper chamber was coated evenly with Matrigel 4 hours prior to cell seeding. Single-cell suspensions of SKOV3, SKOV3-M, SKOV3-L, SKOV3-DDP, SKOV3-DDP-M and SKOV3-DDP-L cells $\left(1 \times 10^{5}\right.$ cells $)$ were placed in the upper chamber, and $600 \mu \mathrm{L}$ of medium containing $15 \%$ FBS was added to the lower chamber as a chemoattractant. After incubation at $37^{\circ} \mathrm{C}$ for 48 hours, the cells that adhered to the lower surface of the membrane were fixed, stained with crystal violet and counted.

\section{Immunohistochemistry (IHC)}

IHC was used to assess the differential expression of ERK in tissues, including 6 pairs of ovarian cancer tissues, normal tissues and tumour tissues from nude mice. All tissues were fixed with 4\% paraformaldehyde, dehydrated, embedded in paraffin, and sectioned. The glass slides were incubated overnight with ERK (9101, Cell Signaling Technology, USA; diluted 1:200), washed three times with PBS, and then incubated with anti-rabbit secondary antibodies (EMD Millipore, Burlington, MA, USA) at $37^{\circ}$ $\mathrm{C}$ for 40 minutes. Finally, the tissue sections were treated with 3',3'-diaminobenzidine until a brown colour developed and counterstained with Harris's modified haematoxylin.The selected proteins were quantified by Image-Pro Plus 6.0 (Media Cybernetics).

\section{Wound Healing Assay}

The migration of treated and untreated ovarian cancer cells was detected by an in vitro wound healing assay. When the cells reached 90-95\% confluence in the 6-well plate, the cell surface was scratched with a $200 \mu \mathrm{L}$ pipette tip, and wound healing was observed under an inverted fluorescence microscope at 0 and 24 hours. The ovarian cancer cells were imaged to ascertain the migration of each group.

\section{Real-Time PCR}

qRT-PCR was used to determine the relative expression levels of LNC00115 and miR-7. miRNA quantification: Bulge-loop $^{\mathrm{TM}}$ miRNA qRT-PCR Primer Sets (one RT primer and a pair of qPCR primers for each set) specific for miR-7 is designed by RiboBio (Guangzhou, China). The sequences of the primers are shown as follow: 5'TGGCTTGTCTTCCATCGTCC-3' (forward) and 5'GCACGAGGGTTGTTACAGGA-3' (reverse) for LINC00115, 5' -ACCACAGTCCATGCCATCAC-3 ' (forward) and 5' - TCCACCCTGTTGCTGTA-3 ' (reverse) for GAPDH. Total RNA was extracted from tissues and cells with TRIzol reagent (Invitrogen, USA), and the SYBR ${ }^{\circledR}$ Green real-time PCR kit (Invitrogen, Carlsbad, CA, USA) was used to detect the expression levels of LNC00115 and miR-7. GAPDH and U6 expression was also quantified and used to determine the relative expression of LNC00115 and miR-7. The relative expression values were normalized and calculated as the fold change in gene expression using the $2^{-\Delta \Delta \mathrm{CT}}$ method.

\section{Nude Mouse Tumour Model}

Thirty-six female 4-week-old BALB/c nude mice were housed under specific pathogen-free conditions (Charles River Laboratories, China). A total of $1 \times 10^{7}$ ovarian cancer cells were injected into the abdominal cavities of the nude 
mice (6 mice per group), and cisplatin was injected intravenously every 3 days at a concentration of $0.4 \mathrm{mg} / \mathrm{mL}$ and an injection volume of $0.1 \mathrm{~mL} / 10 \mathrm{~g}$. The cells injected into mice include SKOV3, SKOV3-M, SKOV3-L, SKOV3-DDP, SKOV3-DDP-M and SKOV3-DDP-L.After 2 weeks of treatment, the mice were sacrificed by cervical dislocation, and tumour tissues were prepared for histological examination. The xenograft tumours were removed, and the tumour volumes (tumour volume $\left(\mathrm{mm}^{3}\right)=0.5 \times$ width ${ }^{2} \times$ length) and weights were calculated. All animal work was performed according to the Guide for the Care and Use of Laboratory Animals, and the related protocols were approved by the Institutional Animal Care and Use Committee of Harbin Medical University First Affiliated Hospital.

\section{Statistical Analysis}

SPSS 22.0 and GraphPad Prism 7.4 were utilized for statistical analyses. All data are presented as the mean \pm standard deviation. Student's $t$ test or one-way analysis of variance was performed to compare two or more groups. Differences were considered statistically significant when $\mathrm{P}<0.05$.

\section{Results \\ LNC00I I5 Regulates Cisplatin Resistance in Ovarian Cancer}

We analysed lncRNA expression in ovarian cancer and noncancerous control tissues using the published dataset GSE14407, revealing that LNC00115 was highly expressed in patients with ovarian cancer (Figure 1A and B). The LNC00115 expression levels in 6 pairs of ovarian cancer and paracancerous tissues were verified by qRTPCR, which showed that LNC00115 was significantly upregulated in OC tissues compared with that in paracancerous tissues. (Figure 1C). Then, we analysed the expression of LNC00115 in SKOV3 and SKOV3-DDP cells by qRT-PCR and found that LNC00115 expression was higher in SKOV3-DDP cells than in SKOV3 cells (Figure 1D).

Based on the above results, we hope to further explore the biological role of LNC00115 in cisplatin resistance in ovarian cancer. We successfully constructed ovarian cancer cell lines in which LNC00115 was knocked out or overexpressed; LNC00115 was overexpressed in SKOV3
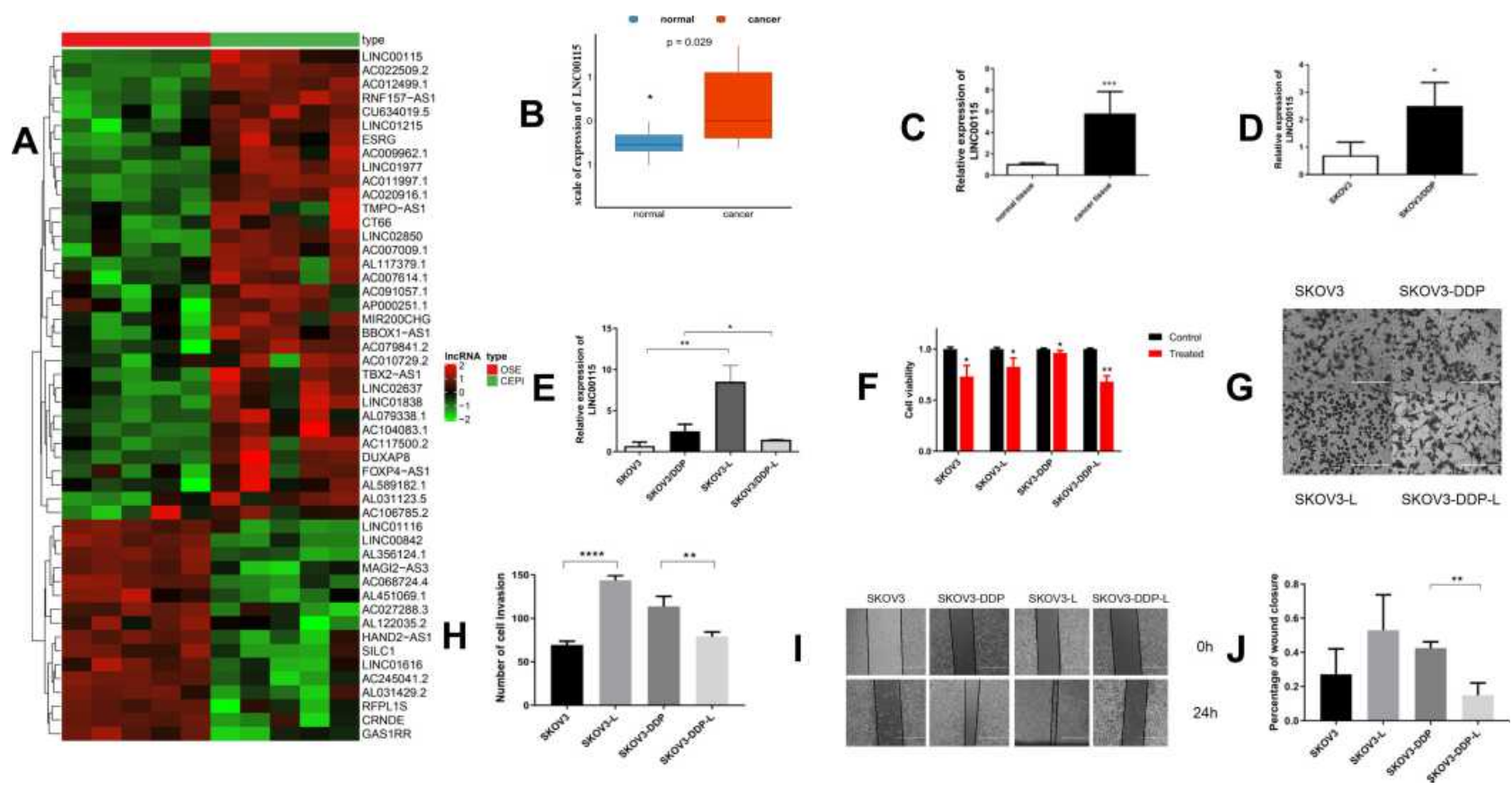

Figure I LNC00II5 regulates cisplatin resistance in ovarian cancer. (A) Hierarchical clustering heat map of differential LNC00II5 expression in ovarian tissues and corresponding normal tissues generated from the GEO database (GSEI4407). The red colour on the heat map denotes upregulation, while green denotes downregulation. (B) The expression of LNC00II5 in an ovarian cancer cohort and normal tissue cohort obtained from the GEO database (GSEI4407). (C) qRT-PCR analysis of LNC00II5 expression in ovarian cancer and paracancerous tissues, $P=0.0002$. (D) qRT-PCR analysis of LNC00I I 5 expression in SKOV3 and SKOV3-DDP cells. (E) qRT-PCR analysis of LNC00II5 expression in SKOV3, SKOV3-DDP, SKOV3-L and SKOV3-DDP-L cells. (F) CCK-8 assays were performed to analyse cell growth after the knockdown or overexpression of LNCO0II5. ( $\mathbf{G}$ and $\mathbf{H}$ ) Cell invasion of ovarian cancer cells treated with different regimens as determined by transwell assays. (I) Representative images of the wound healing abilities of SKOV3, SKOV3-DDP, SKOV3-L, and SKOV3-DDP-L cells. (J) The migration of ovarian cancer cells treated with different regimens as determined by the wound healing assay. Data represent the means $\pm \mathrm{SD}$. $* \mathrm{P}<0.05, * * \mathrm{P}<0.01, * * * \mathrm{P}<0.001$ and $* * * * \mathrm{P}<0.000 \mathrm{I}$. 
cells and knocked out LNC00115 in SKOV3-DDP cells, and its expression was verified by qRT-PCR (Figure 1E). A CCK-8 assay was used to assess the effect of LNC00115 on the cisplatin sensitivity of ovarian cancer cells. Upon treatment with $4 \mu \mathrm{g} / \mathrm{mL}$ cisplatin, the SKOV3-L cells proliferated better than SKOV3 cells (Figure 1F), and the proliferative ability of SKOV3-DDP-L cells was lower than that of SKOV3-DDP cells. Thus, overexpression of LNC00115 enhanced the cisplatin resistance of ovarian cancer cells. Transwell and wound healing assays were used to verify the effect of LNC00115 on the invasion and migration of ovarian cancer cells (Figure $1 \mathrm{G}$ and I). The results showed Overexpression of LNC00115 enhanced the invasion and migration ability of ovarian cancer cells, while knocking down LNC00115 inhibited their invasion and migration abilities (Figure $1 \mathrm{H}$ and $\mathrm{J}$ ). In general, LNC00115 was shown to regulate the development of ovarian cancer cells, and overexpression of LNC00115 enhanced the resistance of ovarian cancer cells to cisplatin.

\section{LNC00II5 Sponges miR-7 in Ovarian Cancer}

Next, we explored the molecular mechanism of LNC00115 in ovarian cancer. It has been indicated that lncRNAs can be used as competing endogenous RNAs (ceRNAs) to regulate the expression of mRNAs by sponging miRNAs. As predicted by TargetScan and miRanda, miR-7 and LNC00115 were found to have complementary sequences. To verify the interaction between these two components, a dual-luciferase assay was performed. Compared with miR-NC, the miR-7 mimics significantly decreased the LNC00115-WT reporter activity but had no effect on that of LNC00115-mut (Figure 2A and B), indicating that LNC00115 can directly target miR-7.

To explore the role of miRNAs in ovarian cancer, we first analysed miRNA expression in ovarian cancer and control tissues using the published dataset GSE53829, revealing that miR-7 expression was significantly lower in the ovarian cancer tissues (Figure 2C and D). Then, the miR7 expression in cisplatin-sensitive and cisplatin-resistant

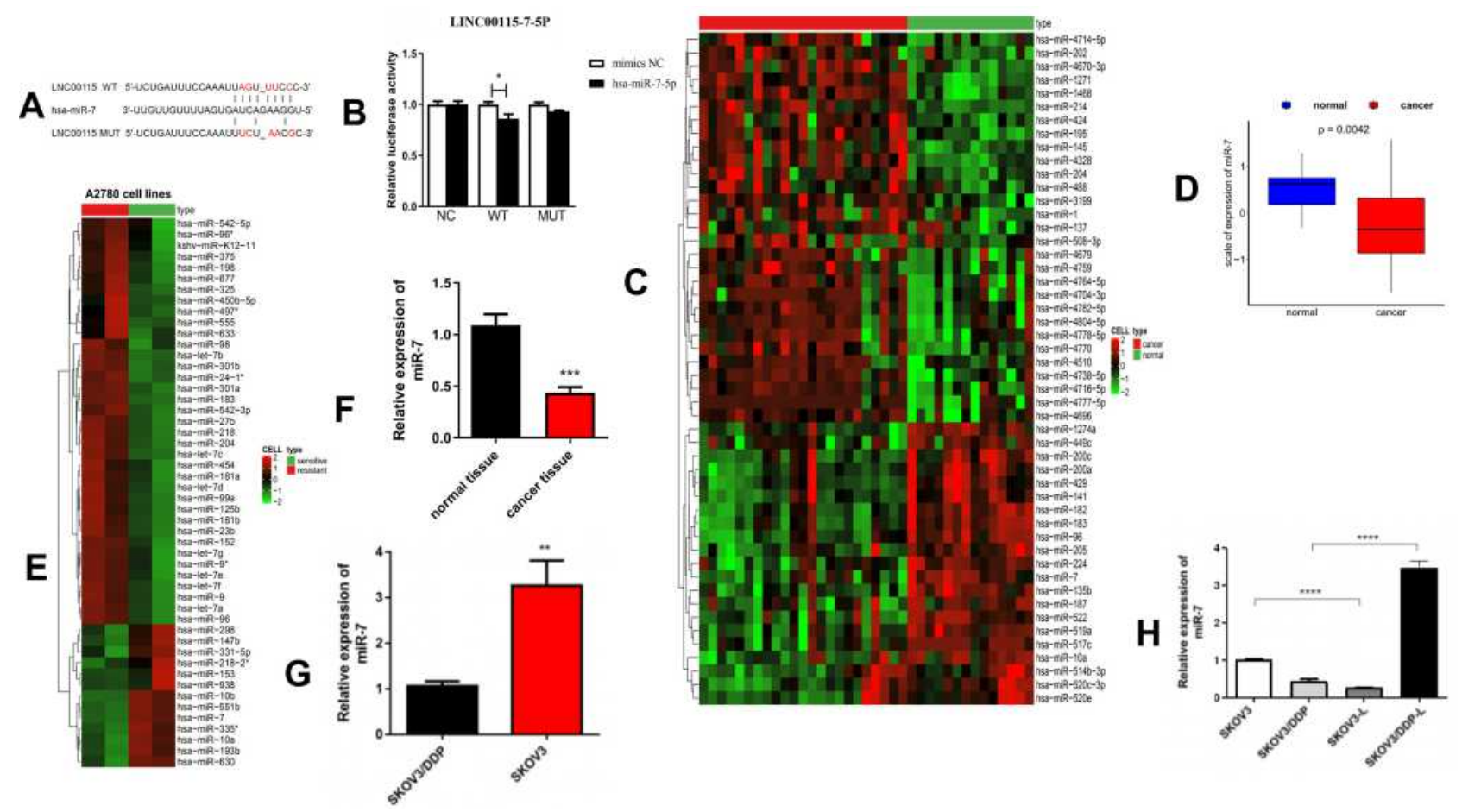

Figure 2 LNC00II5 sponges miR-7 in ovarian cancer cells. (A and B) Dual-luciferase assays were performed to confirm the binding of LNC00II5 to miR-7. (C) Hierarchical clustering heat map of differential miR-7 expression in ovarian tissues and corresponding normal tissues generated from the GEO database (GSE53829). (D) The expression of miR-7 in an ovarian cancer cohort and normal tissue cohort obtained from the GEO database (GSE53829). (E) Hierarchical clustering heat map of differential miR-7 expression in A2780-DDP and A2780 ovarian cells from the GEO database (GSE8420I). (F) qRT-PCR analysis of miR-7 expression in ovarian cancer tissues and normal tissues, $P=0.0007$.(G) qRT-PCR analysis of miR-7 expression in SKOV3-DDP and SKOV3 cells, $P=0.0025$. (H) $q$ RT-PCR analysis of miR-7 expression in SKOV3, SKOV3-DDP, SKOV3-L, and SKOV3-DDP-L cells. Data represent the means \pm SD. $* \mathrm{P}<0.05, * * \mathrm{P}<0.01, * * * \mathrm{P}<0.001$ and $* * * * \mathrm{P}<0.0001$. 
ovarian cancer cell lines was analysed using the published dataset GSE84201, and miR-7 expression was lower in A2780-DDP cells than in A2780 cells (Figure 2E). These data strongly suggest that LNC00115 plays an important role in cisplatin resistance in ovarian cancer by regulating miR-7.

Similarly, we used qRT-PCR to measure the expression of miR-7 in 6 pairs of ovarian cancer and paracancerous tissues, and the results showed that miR-7 was expressed at significantly lower levels in ovarian cancer tissues than in paracancerous tissues (Figure 2F). To further assess whether miR-7 is associated with cisplatin resistance in ovarian cancer, we detected the miR-7 expression in SKOV3 and SKOV3-DDP cells by qRT-PCR, which revealed significantly lower expression in SKOV3-DDP cells than in SKOV3 cells (Figure $2 \mathrm{G}$ ).

To further verify the regulatory effect of LNC00115 on miR-7, we overexpressed LNC00115 in SKOV3 cells and knocked down LNC00115 in SKOV3-DDP cells. The expression of miR-7 in SKOV3, SKOV3-L, SKOV3-DDP and SKOV3-DDP-L cells was detected by qRT-PCR. The expression of miR-7 was significantly lower in SKOV3-L cells than in SKOV3 cells, while the level in SKOV3-DDP -L cells was significantly higher than that in SKOV3-DDP cells (Figure 2H). These results suggest that miR-7 can inhibit the development of ovarian cancer and that its expression is regulated by LNC00115.

\section{Inhibitory Effect of miR-7 on Cisplatin Resistance in Ovarian Cancer}

As mentioned above, miR-7 functions as a suppressor in ovarian cancer. To further verify this role and explore the relationship between miR-7 and cisplatin resistance in ovarian cancer, we overexpressed and knocked out miR-7 in ovarian cancer cell lines, and the expression of miR-7 was verified by qRT-PCR (Figure 3F). We knocked out miR-7 in SKOV3 cells, which showed stronger migration and invasion abilities than SKOV3 cells (Figure $3 \mathrm{~A}$ and $\mathrm{C}$ ). We overexpressed miR-7 in SKOV3-DDP cells, which exhibited significantly worse migration and invasion abilities than the SKOV3-DDP cells (Figure 3B and D). To further determine whether miR-7 affects the cisplatin sensitivity of ovarian cancer, we detected the proliferative ability of SKOV3 and SKOV3-M cells treated with $4 \mu \mathrm{g} / \mathrm{mL}$ cisplatin by the CCK- 8 assay, The proliferative ability of SKOV3-M cells was stronger than that of SKOV3 cells (Figure 3E), while the proliferative ability of SKOV3-DDP-M cells was lower than that of SKOV3-DDP cells. Thus, overexpression of miR-7 reduces cisplatin resistance in ovarian cancer cells, while knocking down miR-7 enhances cisplatin resistance in ovarian cancer cells.
A

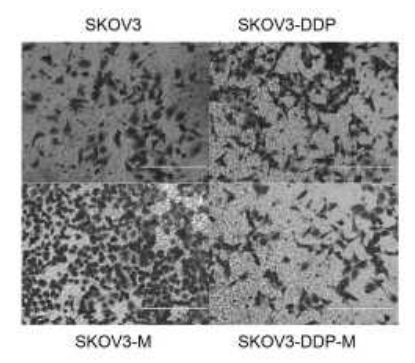

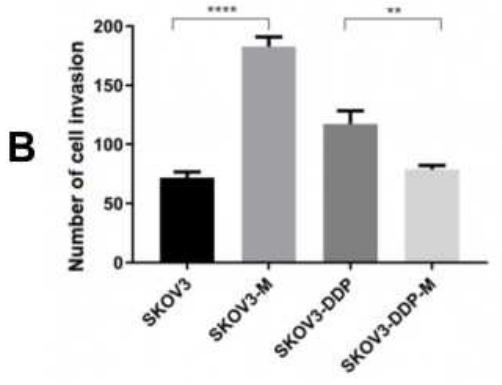

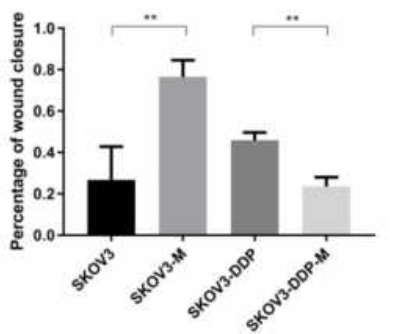

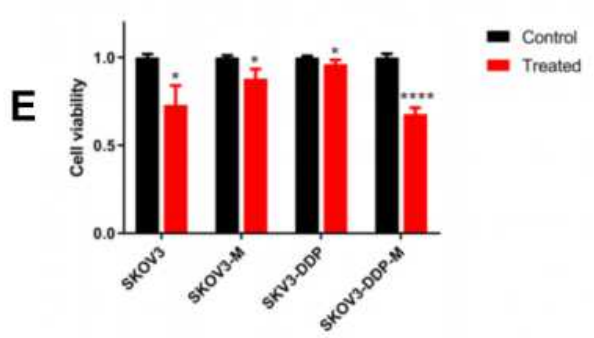

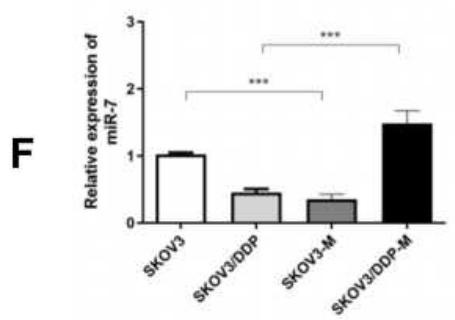

Figure 3 Low miR-7 expression increases the cisplatin resistance, invasion and migration of ovarian cancer cells. (A and B) The invasion of ovarian cancer cells treated with different regimens as determined by transwell assays. (C) Representative images of the wound healing abilities of SKOV3, SKOV3-DDP, SKOV3-M, and SKOV3-DDP-M cells. (D) The migration of ovarian cancer cells treated with different regimens as determined by the wound healing assay. (E) CCK-8 assays were performed to analyse cell growth after the knockdown or overexpression of miR-7. (F) The expression of miR-7 in ovarian cells. Data represent the means $\pm \mathrm{SD}$. $* \mathrm{P}<0.05$, $* * \mathrm{P}<0.0 \mathrm{I}, * * * \mathrm{P}<0.00 \mathrm{I}$ and $* * * * \mathrm{P}<0.0001$. 


\section{LNC00 I I 5 Regulates ERK by Sponging miR-7}

To elucidate the molecular mechanism and target genes of miR-7, we used bioinformatics tools (MiRanda, miRcode) to predict the targets of miR-7, and ERK was identified as a candidate. To further determine the relationship between ERK and miR-7, we confirmed the presence of a functional miR-7 binding site on ERK by a dual-luciferase assay, indicating that ERK is indeed a target gene of miR-7 (Figure 4A and B). Analysis of the published dataset GSE63885 revealed that ERK expression is correlated with the survival of patients with ovarian cancer (Figure $4 \mathrm{C}, P<0$. 05). Six pairs of ovarian cancer and paracancerous tissues were detected by IHC, and the level of ERK was higher in ovarian cancer tissues than in paracancerous tissues (Figure 4E).

To further clarify the mechanisms of ERK and miR-7, we measured ERK protein expression in 6 cell lines by WB and immunofluorescence (Figure 4D, G and H). The expression of ERK in SKOV3-DDP cells was higher than that in SKOV3, SKOV3-DDP-M and SKOV3-DDP-L cells, and the ERK expression in SKOV3 cells was lower than that in SKOV3-M and SKOV3-L cells. Thus, miR-7 and LNC00115 can regulate ERK.

Our results show that LNC00115 affects the cellular ERK expression by regulating the expression of miR-7.

\section{Nude Mouse Tumour Model}

To determine the effect of miR-7 and LNC00115 on the cisplatin resistance of ovarian cancer cells in vivo and the underlying mechanism, $1 \times 10^{7}$ ovarian cancer cells were injected into the abdominal cavities of female nude mice, and tumour growth was monitored throughout 14 days of cisplatin treatment. The tumour weights of the SKOV3-M and SKOV3$\mathrm{L}$ groups were higher than those of the SKOV3 group, while the tumour weights of the SKOV3-DDP group were higher than those of the SKOV3-DDP-M and SKOV3-DDP-L groups. We proved that overexpression of miR-7 or knockdown of LNC00115 can increase the sensitivity of ovarian cancer to cisplatin and inhibit the development of ovarian cancer in vivo (Figure 4F and I). IHC revealed significant differences in ERK expression among the groups of nude mice (Figure 4J). The expression of ERK in SKOV3M-bearing mice was higher than that in SKOV3-bearing mice, and its expression in mice bearing SKOV3-DPP tumours was higher than that in mice bearing SKOV3-DDP-M tumours, which confirmed that miR-7 can regulate the expression of ERK in vivo. The expression of ERK in the SKOV3-L group was higher than that in the SKOV3 group, and its expression in the SKOV3-DDP group was higher than that in the SKOV3-DDP-L group, which confirmed that LNC001155 can regulate the expression of ERK via miR-7 in vivo.

\section{A}

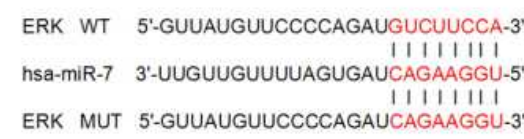
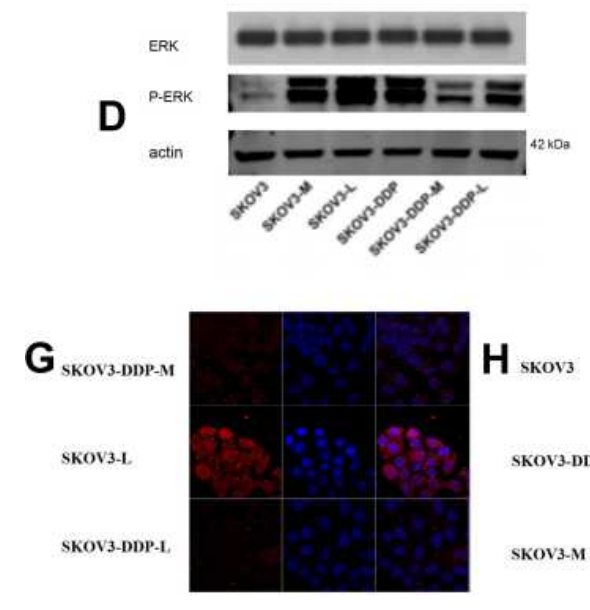

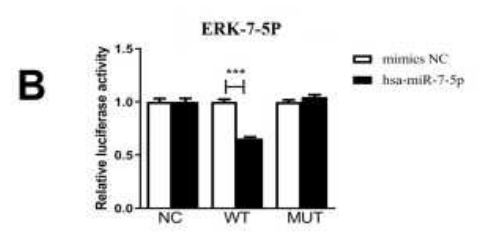

E

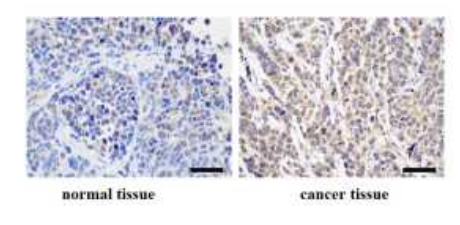

$\mathbf{F}$
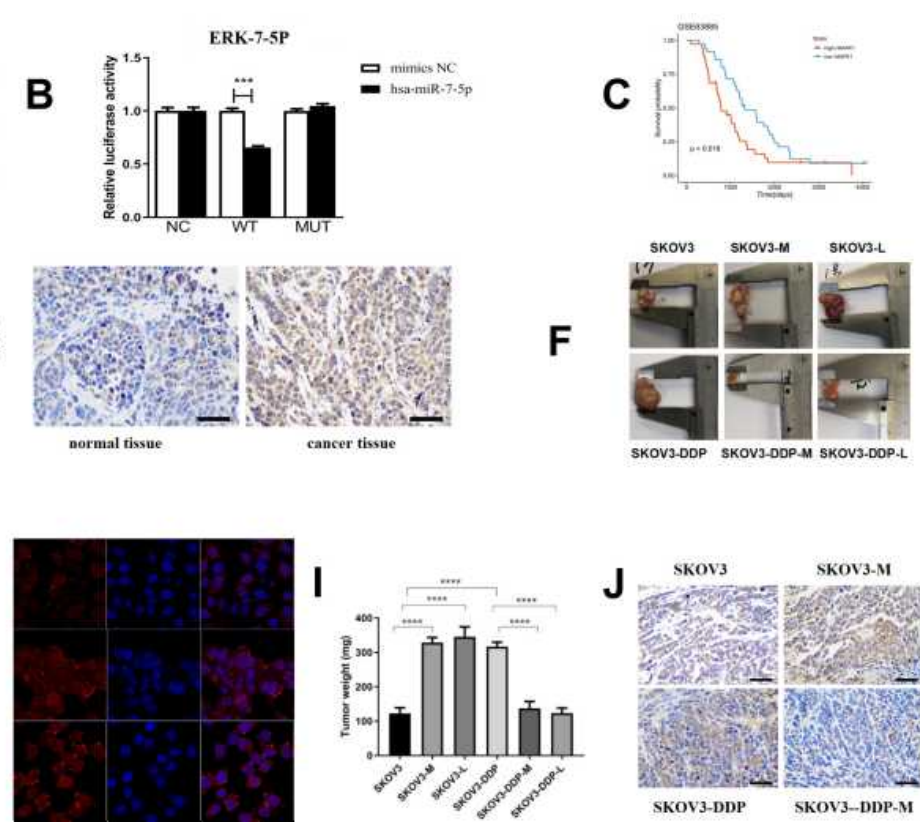

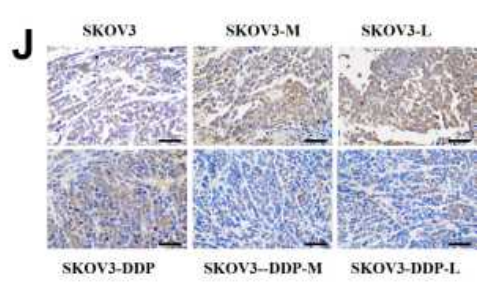

Figure 4 LNC00I I 5 regulates ERK by sponging miR-7. (A and B) Dual-luciferase assays were performed to confirm the binding of ERK to miR-7. (C) The expression of ERK was correlated with the survival of patients with ovarian cancer. (D) p-ERKI/2 and total ERK expression in ovarian cancer cells as determined by WB. (E) ERKI/2 expression in human ovarian tumour tissues and normal tissues as determined by IHC. (F) Nude mouse tumours. (G and $\mathbf{H})$. Immunofluorescence staining of phosphoERKI/2 in ovarian cancer lines. (I) Tumour weights of the nude mice. (J) Detection of ERKI/2 in nude mouse ovarian tumours by IHC. Data represent the means \pm SD. $* * * \mathrm{P}<0.00 \mathrm{I}$ and $* * * * \mathrm{P}<0.000 \mathrm{I}$. 
There is increasing evidence that LNC00115 affects the ERK/MAPK pathway by regulating miR-7, thereby modulating the cisplatin resistance of ovarian cancer cells.

\section{Discussion}

Noncoding RNAs, including lncRNAs, circRNAs, and miRNAs, do not encode proteins but still play important roles in the regulation of gene expression ${ }^{14,15}$ and are related to the occurrence and development of a variety of human diseases. Because of their length and abundant miRNA binding sites, IncRNAs show great potential as markers and therapeutic targets in cancer. Generally, lncRNAs act as oncogenes and have been shown to promote lung cancer, ${ }^{16}$ pancreatic cancer ${ }^{17}$ and epithelial ovarian cancer; ${ }^{18}$ however, they can also act as tumour suppressors (eg, colon cancer). ${ }^{19} \mathrm{~A}$ recent study reported that LNC00115 expression was markedly elevated in colorectal cancer, and LNC00115 was shown to increase the expression of mTOR by inhibiting miR-489-3p expression to promote cell proliferation and migration. ${ }^{20}$ However, its role in ovarian cancer is still unknown. In this study, we confirmed for the first time that LNC00115 is closely related to ovarian cancer. Compared with normal tissues, ovarian cancer tissues exhibited abnormally high LNC00115 expression, suggesting that LNC00115 may be an independent biomarker for the diagnosis of ovarian cancer. Overexpression of LNC00115 significantly promoted the growth, migration and invasion of ovarian cancer cells, and LNC00115 is a potential therapeutic target for the prevention of ovarian cancer metastasis.

Cisplatin chemotherapy is one of the most commonly used methods in the treatment of ovarian cancer. However, approximately $70 \%$ of ovarian cancer patients relapse after receiving cisplatin chemotherapy, ${ }^{21}$ and the mechanism of cisplatin resistance in ovarian cancer is not clear. ${ }^{22}$ In this study, we determined for the first time that LNC00115 is involved in the regulation of cisplatin resistance in ovarian cancer. Compared with that in the ovarian cancer cell line SKOV3, LNC00115 was more highly expressed in the ovarian cancer cisplatin-resistant cell line SKOV3-DDP. Functional analysis in vitro showed that overexpression of LNC00115 significantly increased the cisplatin resistance of SKOV3 cells, while LNC00115 knockdown significantly decreased the cisplatin resistance of SKOV3-DDP cells. The administration of LNC00115 to mice on the standard cisplatin treatment regimen showed a significant promotional effect on ovarian cancer cisplatin resistance in vivo. A series of experiments proved that LNC00115 is a potential target for predicting cisplatin resistance in ovarian cancer.

In addition to elucidating the role of LNC00115 in the diagnosis, prediction and treatment of cisplatin resistance in ovarian cancer, assessing its related molecular mechanism is also very important. Because lncRNAs can be used as miRNA sponges to perform a series of functions, we studied the miRNAs downstream of LNC00115, thereby identifying the candidate gene miR7, and determined the binding site of LNC00115 on miR7 by a dual-luciferase assay. Some studies have shown that miR-7 is related to the occurrence and development of tumours. ${ }^{23}$ For example, miR-7 was shown to inhibit the proliferation and invasion of the human hepatocellular carcinoma cell line SMMC-7721, ${ }^{24}$ and other recent study showed that miR-7 could inhibit the development of ovarian cancer. For example, overexpressed miR-7 can target and inhibit the expression of TGF $\beta 2$, thus inhibiting the growth and metastasis of ovarian cancer cells. ${ }^{25}$ We confirmed the abnormally low expression of miR-7 in ovarian cancer cells. A series of in vitro experiments confirmed that overexpression of miR-7 inhibited the migration and invasion of ovarian cancer cells. miR-7 is not only involved in the diagnosis of ovarian cancer but also related to cisplatin resistance in ovarian cancer. Knocking down miR-7 in SKOV3 cells significantly increased their cisplatin resistance, while overexpression of miR-7 in SKOV3-DDP cells significantly decreased their cisplatin resistance in vitro. In vivo, overexpression of miR-7 was shown to significantly inhibit the cisplatin resistance of ovarian cancer cells. Our results show that miR-7 expression is downregulated in ovarian cancer cells and regulated by LNC00115. In contrast, other study reported higher expression of miR-7 in the serum of patients with ovarian cancer, ${ }^{26}$ and the differential results may be explained by the expression of miR-7 in circulating serum being different from that in ovarian cancer tissues. To address this speculation, we plan to assess the miR-7 expression in circulating sera and ovarian cancer tissues from a large number of patients in the future and to conduct further functional analysis. More importantly, we herein identified that miR-7 is associated with cisplatin resistance in ovarian cancer and may therefore be used as a therapeutic target to combat cisplatin resistance in this gynaecological malignancy in the future. 
The effects of miRNAs are mainly dependent on their downstream targets, and miRNAs can promote or inhibit cancer development and progression by targeting oncogenic or antitumour genes. ${ }^{27}$ We predicted the targets of miR-7 using bioinformatics technology and determined the binding site of miR-7 on ERK by a dual-luciferase assay. The ERK/MAPK pathway regulates many important physiological and pathological processes in cells, such as cell growth, differentiation, ${ }^{28}$ adaptation to environmental stress, and inflammation. ${ }^{29}$ Because the ERK/MAPK pathway plays an important biological role and is highly activated in tumours, inhibition of this pathway is one of the most promising strategies for tumour targeted therapies. An increasing number of studies have shown that abnormal activation of the ERK/MAPK signalling pathway can promote tumour drug resistance. ${ }^{30,31}$ Numerous experiments have proven that ERK is associated with cisplatin resistance in ovarian cancer; for example, MEK/ERK signalling was shown to induce the cisplatin resistance of lung cancer stem cells (CSCs) through the transcriptional activation of GSTP1 expression. ${ }^{32}$ We also found a positive correlation between ERK and LNC00115 expression, and this relationship was shown to be regulated by miR-7. Our study showed that overexpression of miR-7 and knockdown of LNC00115 enhanced the sensitivity of ovarian cancer cells to cisplatin and reduced the proliferation, invasion and migration of ovarian cancer cells. This result may be attributed to deactivation of the ERK/MAPK pathway by miR-7 silencing, thereby enhancing cisplatin-induced cell cycle arrest and apoptosis by activation of the p53/p21 axis. Moreover, miR-7 knockdown was shown to suppress the ERK/MAPK-regulated expression of MMP-2 and MMP-9, the enzymes responsible for degrading the extracellular matrix, and thus decreased the invasion and migration capabilities of the cells. $^{32}$ This conjecture will be verified in future experiments.

Our experiments have shown that LNC00115 can regulate the expression of ERK by sponging miR-7, thereby enhancing the resistance of ovarian cancer cells to cisplatin, and that LNC00115 plays an oncogenic role in the occurrence and development of ovarian cancer. In summary, our findings provide evidence that the LNC00115/ miR-7/ERK axis plays an important role in the cisplatin resistance of ovarian cancer cells, suggesting that alteration of LNC00115 expression is a promising strategy to overcome the chemotherapeutic resistance of ovarian cancer cells to cisplatin.

\section{Conclusions}

Overall, we are the first to confirm that LNC00115 regulates the cisplatin resistance, migration and invasion of ovarian cancer cells via the LNC00115/miR-7/ERK axis.

\section{Ethics Approval and Informed Consent}

The research was approved by the ethics committee of Harbin Medical University (ethics number 2020JS20).

\section{Consent for Publication}

All the authors agree to publish this paper.

\section{Funding}

This work was supported by the National Natural Science Foundation of China (grant number: 81772780).

\section{Disclosure}

The authors report no conflicts of interest in this work.

\section{References}

1. Vaughan S, Coward JI, Bast RC, et al. Rethinking ovarian cancer: recommendations for improving outcomes. Nat Rev Cancer. 2011;11 (10):719-725. doi:10.1038/nrc3144

2. Vargas-Hernández VM, Moreno-Eutimio MA, Acosta-Altamirano G, Vargas-Aguilar VM. Management of recurrent epithelial ovarian cancer. Gland Surg. 2014;3(3):198-202. doi:10.3978/j.issn.2227684X.2013.10.01

3. Atkinson SR, Marguerat S, Bahler J. Exploring long non-coding RNAs through sequencing. Semin Cell Dev Biol. 2012;23 (2):200-205. doi:10.1016/j.semcdb.2011.12.003

4. Tsang WP, Ng EK, Ng SS, et al. Oncofetal H19-derived miR-675 regulates tumor suppressor $\mathrm{RB}$ in human colorectal cancer. Carcinogenesis. 2010;31(3):350-358. doi:10.1093/carcin/bgp181

5. Wang G, Zhang ZJ, Jian WG, et al. Novel long noncoding RNA OTUD6B-AS1 indicates poor prognosis and inhibits clear cell renal cell carcinoma proliferation via the $\mathrm{Wnt} / \beta$-catenin signaling pathway. Mol Cancer. 2019;18(1):15. doi:10.1186/s12943-0190942-1

6. Ta HQ, Whitworth H, Yin Y, et al. Discovery of a novel long noncoding RNA overlapping the LCK gene that regulates prostate cancer cell growth. Mol Cancer. 2019;18(1):113. doi:10.1186/ s12943-019-1039-6

7. Chen C, Luo Y, He W, et al. Exosomal long noncoding RNA LNMAT2 promotes lymphatic metastasis in bladder cancer. $J$ Clin Invest. 2020;130(1):404-421. doi:10.1172/JCI130892

8. Xiong G, Liu C, Yang G, et al. Long noncoding RNA GSTM3TV2 upregulates LAT2 and OLR1 by competitively sponging let-7 to promote gemcitabine resistance in pancreatic cancer. J Hematol Oncol. 2019;12(1):97. doi:10.1186/s13045019-0777-7

9. Lu Y, Zhao X, Liu Q, et al. IncRNA MIR100HG-derived miR-100 and miR-125b mediate cetuximab resistance via $\mathrm{Wnt} / \beta$-catenin signaling. Nat Med. 2017;23(11):1331-1341. doi:10.1038/nm.4424

10. Wang J, Chen J, Sen S. MicroRNA as biomarkers and diagnostics. J Cell Physiol. 2016;231(1):25-30. doi:10.1002/jcp.25056 
11. Qin A, Qian W. MicroRNA-7 inhibits colorectal cancer cell proliferation, migration and invasion via TYRO3 and phosphoinositide 3-kinase/protein B kinase/mammalian target of rapamycin pathway suppression. Int J Mol Med. 2018;42(5):2503-2514. doi:10.3892/ ijmm.2018.3864

12. Yang W, Yang X, Wang X, et al. Silencing CDRlas enhances the sensitivity of breast cancer cells to drug resistance by acting as a miR-7 sponge to down-regulate REG $\gamma$. J Cell Mol Med. 2019;23 (8):4921-4932. doi:10.1111/jcmm.14305

13. Gao Y, Liu J, Huan J, Che F. Downregulation of circular RNA hsa_circ_0000735 boosts prostate cancer sensitivity to docetaxel via sponging miR-7. Cancer Cell Int. 2020;20:334. doi:10.1186/s12935020-01421-6

14. Klingenberg M, Matsuda A, Diederichs S, Patel T. Non-coding RNA in hepatocellular carcinoma: mechanisms, biomarkers and therapeutic targets. $J$ Hepatol. 2017;67(3):603-618. doi:10.1016/j. jhep.2017.04.009

15. McCabe EM, Rasmussen TP. IncRNA involvement in cancer stem cell function and epithelial-mesenchymal transitions. Semin Cancer Biol. 2020. doi:10.1016/j.semcancer.2020.12.012

16. Li G, Li X, Yuan C, et al. Long non-coding RNA JPX contributes to tumorigenesis by regulating miR-5195-3p/VEGFA in non-small cell lung cancer. Cancer Manag Res. 2021;13:1477-1489. doi:10.2147/ CMAR.S255317

17. Liu SL, Cai C, Yang ZY, et al. DGCR5 is activated by PAX5 and promotes pancreatic cancer via targeting miR-3163/TOP2A and activating Wnt/ $\beta$-catenin pathway. Int J Biol Sci. 2021;17(2):498-513. doi:10.7150/ijbs.55636

18. Liu Y, Xu B, Liu M, et al. Long non-coding RNA SNHG25 promotes epithelial ovarian cancer progression by up-regulating COMP. $J$ Cancer. 2021;12(6):1660-1668. doi:10.7150/jca.47344

19. Bian Z, Zhang J, Li M, et al. LncRNA-FEZF1-AS1 promotes tumor proliferation and metastasis in colorectal cancer by regulating PKM2 signaling. Clin Cancer Res. 2018;24(19):4808-4819. doi:10.1158/ 1078-0432.CCR-17-2967

20. Feng W, Li B, Wang J, et al. Long non-coding RNA LINC00115 contributes to the progression of colorectal cancer by targeting miR-489-3p via the PI3K/AKT/mTOR pathway. Front Genet. 2020;11:567630. doi:10.3389/fgene.2020.567630

21. Giornelli GH. Management of relapsed ovarian cancer: a review. Springerplus. 2016;5(1):1197. doi:10.1186/s40064-016-2660-0

22. Li J, Pan C, Boese AC, et al. DGKA provides platinum resistance in ovarian cancer through activation of c-JUN-WEE1 signaling. Clin Cancer Res. 2020;26(14):3843-3855. doi:10.1158/1078-0432.CCR19-3790
23. Xin L, Liu L, Liu C. DNA-methylation-mediated silencing of miR-7-5p promotes gastric cancer stem cell invasion via increasing Smo and Hes1. J Cell Physiol. 2020;235(3):2643-2654. doi:10.1002/ jcp. 29168

24. Zhang ZY, Zheng YP, Dong KS, Dong HH. Effect of microRNA-7 on proliferation, invasion, migration and EMT of hepatoma cell line SMMC-7721. Eur Rev Med Pharmacol Sci. 2020;24 (23):12153-12160. doi:10.26355/eurrev_202012_24004

25. Gu Y, Zhang S. High-throughput sequencing identification of differentially expressed microRNAs in metastatic ovarian cancer with experimental validations. Cancer Cell Int. 2020;20:517. doi:10.1186/s12935-020-01601-4

26. Meng X, Joosse SA, Müller V, et al. Diagnostic and prognostic potential of serum miR-7, miR-16, miR-25, miR-93, miR-182, miR-376a and miR-429 in ovarian cancer patients. Br J Cancer. 2015;113(9):1358-1366. doi:10.1038/bjc.2015.340

27. Ma Y, Sun Y. miR-29a-3p inhibits growth, proliferation, and invasion of papillary thyroid carcinoma by suppressing NF- $\kappa \mathrm{B}$ signaling via direct targeting of OTUB2. Cancer Manag Res. 2019;11:13-23. doi:10.2147/CMAR.S184781

28. Vishnu VV, Muralikrishna B, Verma A, et al. C3G regulates STAT3, ERK, adhesion signaling, and is essential for differentiation of embryonic stem cells. Stem Cell Rev Rep. 2021. doi:10.1007/ s12015-021-10136-8

29. Huang X, Yu P, Liu M, et al. ERK inhibitor JSI287 alleviates imiquimod-induced mice skin lesions by ERK/IL-17 signaling pathway. Int Immunopharmacol. 2019;66:236-241. doi:10.1016/j. intimp.2018.11.031

30. Li J, Ye T, Liu Y, et al. Transcriptional activation of Gstp1 by MEK/ ERK signaling confers chemo-resistance to cisplatin in lung cancer stem cells. Front Oncol. 2019;9:476. doi:10.3389/fonc.2019.00476

31. Kulshrestha A, Katara GK, Ibrahim SA, et al. Targeting V-ATPase isoform restores cisplatin activity in resistant ovarian cancer: inhibition of autophagy, endosome function, and ERK/MEK pathway. J Oncol. 2019;2019:2343876. doi:10.1155/2019/2343876

32. Yu T, Bai W, Su Y, Wang Y, Wang M, Ling C. Enhanced expression of lncRNA ZXF1 promotes cisplatin resistance in lung cancer cell via MAPK axis. Exp Mol Pathol. 2020;116:104484. doi:10.1016/j. yexmp.2020.104484

\section{Publish your work in this journal}

Cancer Management and Research is an international, peer-reviewed open access journal focusing on cancer research and the optimal use of preventative and integrated treatment interventions to achieve improved outcomes, enhanced survival and quality of life for the cancer patient.
The manuscript management system is completely online and includes a very quick and fair peer-review system, which is all easy to use. Visit http://www.dovepress.com/testimonials.php to read real quotes from published authors. 